(c) American Dairy Science Association, 2006.

\title{
A Model for Detection of Individual Cow Mastitis Based on an Indicator Measured in Milk
}

\author{
M. G. G. Chagunda, ${ }^{1}$ N. C. Friggens, M. D. Rasmussen, and T. Larsen \\ Department of Animal Health, Welfare and Nutrition, Danish Institute of Agricultural Sciences, PO Box 50, DK-8830 Tjele, Denmark
}

\begin{abstract}
A dynamic deterministic biological model was developed that generates, for a given cow on a given day, a value for her risk of having mastitis. The model combines real-time information from a mastitis indicator measured in milk with additional factors that are other known risk factors of mastitis but that are not reflected in the indicator. L-Lactate dehydrogenase (LDH), an enzyme whose activity is increased because of mastitis, is used as an example of a mastitis indicator. The additional factors incorporated in the model are days from calving, breed, parity, milk yield, udder characteristics, other disease records, electrical conductivity, and herd characteristics. The model is designed to run each time a new $\mathrm{LDH}$ value is recorded and can run in the absence of the additional factors. Electrical conductivity measurements and disease records, where available, also trigger the model to run. As an input, milk LDH activity values $(\mu \mathrm{mol} / \mathrm{min}$ per $\mathrm{L}$ ) are multiplied by milk yield (L) to produce the amount of $\mathrm{LDH}(\mu \mathrm{mol} / \mathrm{min})$ and are then smoothed using an extended Kalman filter before being processed by the biological model. The output comprises a risk of acute mastitis and a relative degree of chronic mastitis. The model also produces a days-tonext sample value that allows sampling frequency to be either increased or reduced depending on the risk of mastitis. The days-to-next sample value was designed to make the best use of opportunities afforded by automated, inline sampling technology. The model functionality was investigated using simulated data, and real-farm data of naturally occurring mastitis were then used to validate the model. The results demonstrated that the model is robust to sampling frequency and random noise in the $\mathrm{LDH}$ measurements. It was able to detect mastitis reasonably well: Using a threshold mastitis risk of 0.7 , sensitivity for detecting clinical mastitis was $82 \%$. Specificity, that is, the ability to avoid misclassifying healthy observations as mastitis, was $99 \%$.
\end{abstract}

Received October 27, 2005.

Accepted February 28, 2006.

${ }^{1}$ Corresponding author: Mizeck.Chagunda@agrsci.dk
Key words: biological model, mastitis, risk, indicator in milk

\section{INTRODUCTION}

Mastitis is one of the major causes of serious economic losses, animal suffering, negative effects on milk quality, and reduced product hygiene. Sustainable milk production should therefore not only aim at effective treatment of mastitis but also at implementing efficient prevention measures. However, effective mastitis control strategies need to be based on early and accurate detection. Early detection has many potential benefits, 2 of which are that it allows proactive management strategies to be implemented to abrogate negative effects of disease (Fricke, 2002) and that it leads to better cure rates (Deluyker et al., 2005). However, this is becoming increasingly difficult to achieve because of the generally increasing trend in herd size, coupled with an associated increase in the number of cows per husbandry person. The result has been that less time and attention is being devoted to each cow (Lucy, 2001). One way to overcome this problem is through the use of automated mastitis monitoring. Advances in biosensor technology, together with the advent of inline automated milk sampling and processing systems, have made on-farm automated real-time analysis of milk-based mastitis indicators a realistic proposition (Frost et al., 1997; Mottram et al., 2002). However, it is generally agreed that no single indicator captures all aspects of mastitis (Hamann, 2005). This is especially so if the aim is to achieve early detection. It is our view that early detection of mastitis can best be achieved by combining important information from a real-time indicator with other known risk factors. To do this, a model is required.

A number of mastitis detection models have been developed previously with varying degrees of accuracy and automation-for example, models based on electrical conductivity (Cond), milk yield, and milk temperature (de Mol et al., 1999; de Mol and Ouweltjes, 2001; de Mol and Woldt, 2001). However, models are currently lacking that combine time-series measurements of an indicator with other known mastitis predisposing factors of direct physiological relevance to the status of udder health throughout the lactation. The objective of 
the current study was to develop a biological model for early detection of mastitis for individual cows in a dairy herd based on inline real-time measurements of an indicator in milk and additional mastitis risk factors. Such a model, with focus on early mastitis detection, allows timely follow-up and thus more targeted prevention and treatment strategies. This paper presents a model using the milk enzyme L-lactate dehydrogenase (LDH) as an indicator, although in principle the model would apply to any mastitis indicator measured in milk.

\section{MODEL DESCRIPTION}

\section{Overview of the Model}

The model is dynamic and deterministic, designed to run each time a new trigger input occurs, and the system is hybrid in nature. Hybrid systems calculate their values by combining continuous-time dynamics, discrete events, and discrete mode changes. The major inputs to the model are LDH measurement, milk yield, and calving date. L-Lactate dehydrogenase measurements are used to generate an indicator-based risk (MIBR), whereas other animal- and herd-related factors generate an additional risk factor (ARF). Together, $M I B R$ and $A R F$ are used to generate an overall risk of mastitis. This structural separation reflects the underlying logic that $A R F$ are only those factors whose effects are not acting on LDH activity. The model makes the distinction between acute and chronic cases of mastitis. An acute mastitis incident is characterized by a rapid or sudden increase in the amount of LDH over a relatively short period, whereas chronic mastitis is characterized by a high but gradual increase in the amount of $\mathrm{LDH}$ over a long period.

The outputs of the model are 1) the overall risk of acute mastitis (AcuteRisk), 2) the relative degree of chronic mastitis (ChronDeg), and 3) a calculation of when to take the next sample (days to next sample; DNS), which is designed to feed back to the sampling device. In the model, the 3 outputs are generated by separate equations. Apart from the inputs, the outputs, and the equations described hereafter, the model includes a set of constants, the values for which are given in Tables 1 and 2 . The constants are based on the literature and on expert opinion.

Sigmoid functions of the Gompertz form, $y=$ $\exp \{-\exp [R(x-T)]\}$, are widely used in the model because they allow the transition between 0 and 1 to be modeled using only 2 coefficients, $R$ and $T$, which are easily interpretable ( $x$ is usually time). In the Gompertz function, $T$ gives the point of inflection of the curve, at which the transition between 0 and 1 is approximately one-third. (For the curve to be sigmoid, $T$ must fall within the range of $x$ values.) $R$ is the rate coefficient.
If $R$ is positive, then the function is decreasing from 1 to 0 as $x$ increases. If $R$ is negative, then the function is increasing from 0 to 1 as $x$ increases. The larger is $R$, the more extreme is the S-shape. Large values of $R$ allow a virtually binary response to be modeled (either 0 or 1 with nothing in between), whereas very small values of $R$ result in a virtually linear transition from 0 to 1 . Gompertz functions have been widely used in biological systems because they characterize some underlying physiological or biochemical mechanisms whose effectiveness decays with time (France and Thornley, 1984). These functions also have the property that, regardless of the range in $x$, the outcome, $y$, is always bounded between 0 and 1 . This provides an elegant way to limit ranges.

\section{Model Inputs}

The model is based on the following inputs: cow identification number, number of days from calving (calving date), parity, LDH, milk yield, udder characteristics, herd-level mastitis, disease recordings, and Cond where available. Not all inputs trigger a new run of the model; the model is triggered by LDH, Cond, and disease incidences. The time of occurrence associated with each of the inputs is required. Cow identification number, number of days from calving, and parity are self-evident and are not discussed further. The product of $\mathrm{LDH}$ and milk yield is the main component of $M I B R$, whereas milk yield acceleration (MYAcc), udder characteristics, herd-level mastitis, disease recordings, and Cond are the main components of $A R F$. The elements of the 2 overall inputs, $M I B R$ and $A R F$, are described in detail in the following sections.

\section{Elements of MIBR}

The MIBR is based on measurements of LDH activity in milk ( $\mu \mathrm{mol}$ of product/min per L). A strong, positive correlation has been found between LDH activity and SCC in milk samples from clinically mastitic cows (Bogin et al., 1977; Harmon, 1994). Our own study of LDH activity, measured at every milking from 197 cows for a period of $8 \mathrm{mo}$, showed a correlation of 0.8 between $\mathrm{LDH}$ activity and SCC in naturally occurring mastitis and 0.5 in healthy cows (Chagunda et al., 2006). An important issue for the use of milk measurements, including LDH, concerns the effects of dilution. Assuming that the amount of LDH activity ( $\mu \mathrm{mol} / \mathrm{min}$ ) is a function of the amount of epithelial and somatic cell damage (Bogin et al., 1977; Kitchen et al., 1980; Kato et al., 1989 ), then the measured LDH activity ( $\mu \mathrm{mol} / \mathrm{min}$ per $\mathrm{L}$ ) will depend on milk yield. In addition, mastitis usually occurs in only one quarter at a time, and the milk 
Table 1. Values for the constants used in the biological model other than those related to disease

\begin{tabular}{|c|c|c|}
\hline Constant & Value & Description \\
\hline DNSdef & 5 & Default days to next sample \\
\hline StableInt ${ }^{1}$ & 7 & Time interval over which Stable is calculated (d) \\
\hline MaxSlope & 24 & Scalar for RiskSlope \\
\hline MaxLevel & 60 & Scalar for RiskLevel \\
\hline $\operatorname{MaxAcc^{2}}$ & 3 & Scalar for milk yield acceleration (L/d per $\mathrm{d}$ ) \\
\hline MaxMDur & 0.4 & Maximum risk due to milking duration \\
\hline MDurR & -0.008 & Rate for milk duration risk \\
\hline MDurT ${ }^{3}$ & 400 & $T$ value for milk duration risk \\
\hline$M a x P M F$ & 0.3 & Maximum risk due to milk flow \\
\hline$P M F R$ & -0.2 & Rate for milk flow risk \\
\hline$P M F T^{3}$ & 55 & $T$ value for milk flow risk $(\mathrm{mL} / \mathrm{s})$ \\
\hline MaxCond & 3.0 & Scalar for conductivity \\
\hline$A R F W$ & 0.25 & Additional risk factor $(A R F)$ weighting constant \\
\hline SpreadR & -0.04 & Rate value for spread of bacteria due to cow density \\
\hline SpreadT $T^{3}$ & 50 & $T$ value for spread of bacteria due to cow density \\
\hline BacLifeMax & 0.005 & Maximum value of bacteria life (wk) \\
\hline$Z M$ & 3 & Adjustment factor for conductivity \\
\hline Short $T^{3}$ & 0.1 & Associated risk increase due to short teats \\
\hline LowUdd & 0.05 & Associated risk increase due to low udder \\
\hline Leaky $T^{3}$ & 0.15 & Associated risk increase due to leaky teats \\
\hline
\end{tabular}

from that quarter is diluted by milk in the other 3 quarters. A further complication is that the ratio of the amounts of milk produced by the infected and healthy quarters is also affected by mastitis. To minimize these effects, and especially because it is envisaged that inline milk measurements will be made at the cow level rather than at the quarter level, the indicator variable in the model is the amount of $\mathrm{LDH}(\mu \mathrm{mol} / \mathrm{min})$, which is the product of $\mathrm{LDH}$ activity $(\mu \mathrm{mol} / \mathrm{min}$ per $\mathrm{L})$ and milk yield (L).

Filtering and Smoothing the LDH Amount Time Series. To reduce random noise in the time series, the amounts of LDH used in the biological part of the model are smoothed values. A biometric module was used to generate these smoothed values. This was an extended Kalman filter, using a local linear growth model with outliers (e.g., Smith and West, 1983). The smoothed values (called Level, $\mu \mathrm{mol} / \mathrm{min}$ ) are posterior mean estimates of the true level in this model. The assumptions made in the local linear growth model, the estimation of parameters, and the implementation of the extended Kalman filter are described in Korsgaard and Løvendahl (2002). In brief, this being a dynamic linear model for time-series data, it estimates the state of any new

Table 2. Constants ${ }^{1}$ used in the model for each disease when present as an additional risk factor for mastitis

\begin{tabular}{|c|c|c|c|c|}
\hline Disease & Class $^{2}$ & $\operatorname{MaxDis}^{3}$ & $D i s R^{4}$ & $D i s T^{5}$ \\
\hline Metritis & Infection & 0.5 & 0.4 & 10 \\
\hline Teat injury & Infection & 0.9 & 0.4 & 21 \\
\hline Acidosis $^{6}$ & Infection & 0.5 & 0.4 & 14 \\
\hline Ketosis & Noninfection & 0.2 & 0.2 & 21 \\
\hline Milk fever & Noninfection & 0.2 & 0.4 & 10 \\
\hline Retained placenta & Noninfection & 0.4 & 0.4 & 10 \\
\hline
\end{tabular}

\footnotetext{
${ }^{1}$ Values determined from the literature and a panel of experts, as described in the Overview of the Model section.

${ }^{2}$ The diseases classified as "infection" in the model are basically those that are associated with a potentially increased bacteria load in the cow, whereas those classified as "noninfection" are related to metabolic problems, without a high bacteria load.

${ }^{3} \mathrm{MaxD}$ is gives the effect of each disease on the additional risk factor on the day of occurrence, as applied in Equation 1.

${ }^{4} \mathrm{DisR}$ is a rate coefficient indicating the rate of decay of the disease effect from the time of occurrence.

${ }^{5} \mathrm{DisT}$ is the point of inflection of the curve giving the decay of the disease effect from the time of occurrence.

${ }^{6}$ Acidosis is included in the infection class because of its effect on endotoxins in the bloodstream.
} 
milk LDH value based on data from the previous time step and the current measurement. Hence, any new data point may belong to 1 of the 4 groups: normal evolution, outlier, slope change, and level change. The biometric model generates estimates of the probability that any given $\mathrm{LDH}$ observation belongs to one of these groups and smooths the time series accordingly. The prior probabilities of belonging to a particular group and the variances associated with the different models, as well as the parameters associated with the initial state, were estimated from a data set of daily milk $\mathrm{LDH}$ values collected from 100 cows over a 6 -mo period. These parameters were treated as known when producing Level parameters of LDH. The extended Kalman filter generates smoothed values of varying accuracy, depending on the degree of back smoothing used. Zerostep back smoothing provides posterior mean estimates of $\mathrm{LDH}$ activity level, that is, estimates of LDH activity level at time $t$ from samples up to and including time $t$. One-step-back smoothing provides estimates of $\mathrm{LDH}$ activity level at time $t-1$ from samples up to and including time $t$. Clearly, using back-smoothed values implies accepting a lag in availability of level estimates (producing a one-step-back-smoothed value requires a subsequent sample to have been taken). This process can, in principle, be extended with 2-step smoothing, 3 -step smoothing, and so on back. However, this was not considered because the gains in accuracy diminish relative to the associated lag in availability. Because the optimal trade-off between time lag in availability of smoothed values and accuracy of the smoothed values may vary according to local conditions (e.g., frequency of milk sampling), the biometric module was implemented so that either zero- or one-step-back-smoothed values could be used in the biological component of the model. The rate of change in Level, LDH slope (Slope, $\mu \mathrm{mol} / \mathrm{min}$ per d), was calculated from the difference between consecutive Level values using a 3-d rolling average with exponential weighting.

To distinguish between the short-term changes in the amount of LDH symptomatic of acute mastitis and the long-term changes associated with chronic mastitis, the underlying trend in the amount of $\mathrm{LDH}$ is calculated. This underlying trend in the amount of LDH is called Stable. Stable is calculated as a rolling average of Level $(\mu \mathrm{mol} / \mathrm{min}$ ) for each cow over an interval of $7 \mathrm{~d}$. A default value of Stable at calving is needed to calculate the risk due to Level. The default Stable value is set low $(2 \mu \mathrm{mol} /$ min) and is diluted out as actual Level data accumulates.

Calculating MIBR. As shown in Figure 1, MIBR is the sum of the risk due to Level (RiskLevel) and the risk due to Slope (RiskSlope). The assumption in the model is that the greater the Slope, the greater the risk of acute mastitis. The equation for calculating the risk due to slope is

$$
\begin{gathered}
\text { RiskSlope }=\text { Slope }(1+\text { signSlopeChan } \\
\times \text { PSlope }) / \text { MaxSlope }
\end{gathered}
$$

where SignSlopeChan gives the sign of the slope change (current Slope - previous Slope), returning a value of +1 for slope changes $\geq 0$ and -1 for slope changes $<0$; PSlope is the probability of a slope change calculated by the extended Kalman filter in the biometric module; MaxSlope is a constant giving the value of the slope that will give a risk of 1 . The term signSlopeChan $\times$ PSlope prevents RiskSlope from being increased by a high probability of a slope change when the associated change in slope is negative (i.e., PSlope only increases RiskSlope when Slope is increasing).

Risk Due to LDH Level (Level). It is assumed that the higher the Level relative to the stable level (Stable), the greater the risk of acute mastitis. Stable is used as a baseline to facilitate the differentiation between acute and chronic mastitis; it assumes that the increase in LDH caused by an acute case is independent of the underlying stable level. The equation for the RiskLevel is

$$
\begin{aligned}
\text { RiskLevel }= & (\text { Level }- \text { Stable }(1+\text { signLevChan } \\
& \times \text { PLevel }) / \text { MaxLevel }
\end{aligned}
$$

where signLevChan gives the sign of the level change (Level - LastLev), returning a value of +1 for level changes $\geq 0$ and -1 for the level changes $<0$, PLevel is that probability of a level change calculated by the extended Kalman filter, and MaxLevel is a constant. LastLev is the value of Level from the preceding model run. Level - Stable is not allowed to be negative.

\section{Elements of ARF}

When considering which factors to include as $A R F$ for mastitis, an important criterion was that the risks accounted for by $A R F$ were not already included in the $M I B R$ - that is, any factor that directly affects LDH has not been included as an $A R F$. In the event that a potential risk factor has an effect on $\mathrm{LDH}$ and also an additional effect, the 2 effects are distinguished and only the additional effect of the factor is included in the $A R F$.

Having identified those aspects of mastitis biology (e.g., teat canal defenses, infection pressure) that should be included, a further important issue in implementing these ARF was that they should be based on measures that can reasonably be expected to be avail- 


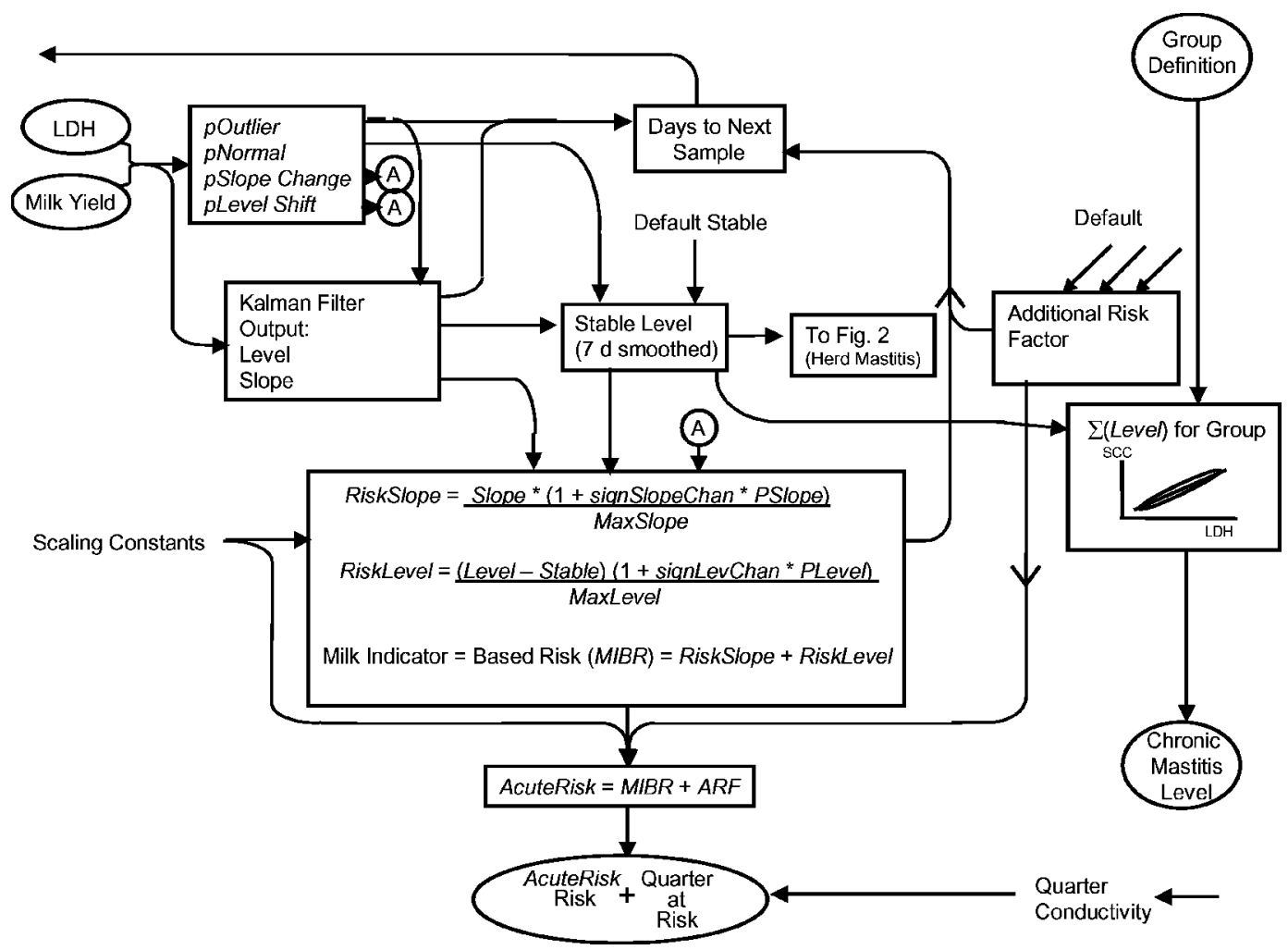

Figure 1. Model architecture for the indicator-based risk (MIBR) part of the mastitis model. pOutlier, pNormal, pSlope Change, and pLevel Shift give the probability that the L-lactate dehydrogenase (LDH) measurement belongs to classes: outlier, normal evolution, a slope change, and a level shift. Level is the Kalman filter output amount of LDH, Slope is the rate of change in Level. Stable is calculated as an average of Level ( $\mu \mathrm{mol} / \mathrm{min}$ ) for each cow over an interval of $7 \mathrm{~d}$. RiskSlope and RiskLevel are risks of acute mastitis due to either slope change or level change, respectively. AcuteRisk is the sum of MIBR and additional risk factors (ARF).

able on farm now (or in the near future). For model to be of practical use, in some cases we have used inputs that are not state-of-the-art, thereby retaining applicability. The $A R F$ included in the current model are $M Y$ $A c c$, milking duration, udder characteristics, herd mastitis level, current lactation disease history, and quarter level conductivity. Details of these factors are described in the following sections. The $A R F$ model architecture is presented in Figure 2.

MYAcc. Acceleration in milk yield (L/d per d) is a way of combining milk yield and days from calving, which crystallizes the components of these 2 factors into an index that is designed to reflect the physiological stress being experienced by the cow. The rate at which an animal performs, either in absolute terms or relative to a potential maximum, is generally agreed to be an important factor in determining that animal's ability to cope with metabolic load (Kronfeld, 1976; Knight et al., 1999). However, the majority of stress-induced diseases occur substantially earlier than the peak (rate) of milk production. Ingvartsen et al. (2003) argued that $M Y A c c$, which is maximal just after calving, may therefore be a better predictor of physiological stress. It is also greater in high-yielding cows. Thus, MYAcc indexes 2 major risk factors for mastitis without directly using milk yield, which is a component of the amount of $\mathrm{LDH}$ and hence is reflected in the MIBR. In the current model, MYAcc is derived from milk yield values smoothed using the Kalman filter. The reason for this is that MYAcc is being used as an indicator of underlying physiological status; thus, it is the underlying biological trend that is of value, not short-term day-to-day fluctuations. This process is akin to accepted procedures used to derive milk yield curve components (e.g., Friggens et al., 1999) or conductivity measures (e.g., Woolford et al., 1998). The equation used to determine risk of mastitis (RiskAcc) due to MYAcc is

$$
\text { RiskAcc }=\text { MYAcc/MaxAcc }
$$

where MaxAcc is a scaling constant that gives the level of acceleration, which will return a risk of 1 .

Duration of Milking. Duration of milking (MilkDur) is used as an index reflecting the negative effect of machine milking on teat integrity. The underlining assumption is that the longer the duration, the higher 


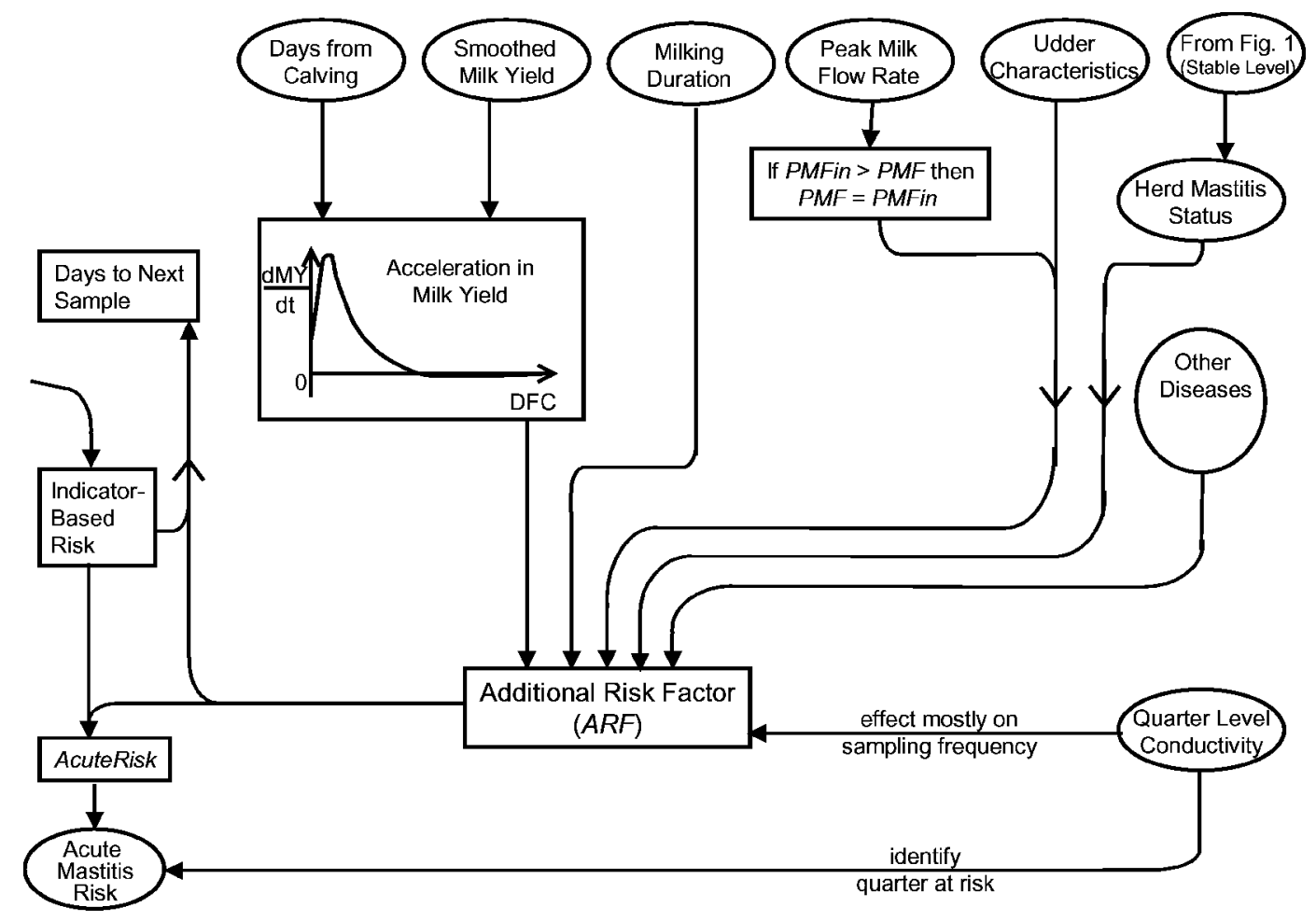

Figure 2. Model architecture for the additional risk factor $(A R F)$ part of the mastitis model. PMFin is the measured value of peak milk flow, $P M F$ is the default value of peak milk flow. Stable level is calculated as an average of Level ( $\mu$ mol/min) for each cow over an interval of $7 \mathrm{~d}$ (coming from Figure 1). DFC = days from calving.

the risk of mastitis. This assumption has been confirmed by several researchers: Machine milking can result in congestion and edema of the teat tissue, especially at the teat end, and can also influence teat diameter, penetrability of the teat canal, and teat defense mechanisms (Bramley and Dodd, 1984; Hamann and Duck, 1984). Further, machine milking can transmit pathogens, both passively from the teat skin of one cow to the teat cistern of another, and actively by dispersing them inside the udder (Hamann, 1987). In the model, the risk of mastitis due to duration of milking (RiskMilkDur) is calculated as

$$
\begin{gathered}
\text { RiskMilkDur = MaxMDur } \times \exp \\
\{-\exp [\text { MDurR }(\text { MilkDur }- \text { MDurT })]\}
\end{gathered}
$$

where MilkDur is the duration of milking (s), and MaxMDur, MDurR, and MDurT are constants. Given the constant values in Table 1, RiskMilkDur increases from 0.011 to 0.24 and 0.37 as MilkDur increases from 240 to 480 and $720 \mathrm{~s}$ (the maximum risk, given by MaxMDur, is 0.4).

Udder Characteristics. The purpose of these inputs is to index the cow's inherent susceptibility to mastitis caused by the physical characteristics of the udder that relate to teat integrity (Hamann, 1987). Included in the model are teat length, udder depth, teat leakiness, and teat canal diameter. Teat length, udder depth and teat leakiness are handled as binary traits. Thus, the user can input short teats, low udder, and leaky teats (LeakyT). These increase the risk of mastitis attributable to udder characteristics (RiskUdd) by $0.1,0.05$, and 0.15 , respectively (Table 1 ). Because these characteristics are not expected to change on a short time scale, once input they are retained for the rest of the lactation. If a given cow is not recorded as having short teats (ShortT), then Short T = 0, but if she is recorded as having ShortT, then Short T takes the value given in Table 1. The same applies to LowUdd and LeakyT.

Teat canal diameter is not a measure that is commonly made on farm; therefore, we have chosen to use peak milk flow rate (PeakMF) as a proxy for teat canal diameter. Teat canal diameter does not change markedly through lactation, although PeakMF varies with milk yield (Baxter et al., 1950; Giesecke et al., 1972). To minimize disturbance caused by milk yield, we chose to use the maximum recorded PeakMF within a given lactation. This is obtained by retaining the maximum recorded PeakMF as the lactation progresses. A default maximum PeakMF of $50 \mathrm{~mL} / \mathrm{s}$ is assumed at the start 
of lactation. The retained maximum recorded PeakMF is used to calculate the associated risk. This has the practical advantage that a value is available from the start of lactation. Because this routine is vulnerable to noisy measurements, the input PeakMF used are the smoothed outputs of the Kalman filter. The PeakMF $(\mathrm{mL} / \mathrm{s})$ is converted to a risk factor (PeakMFRisk) by the following equation:

$$
\begin{gathered}
\text { PeakMFRisk }=\text { MaxPMF } \times \exp \\
\{-\exp [P M F R(P e a k M F-P M F T)]\}
\end{gathered}
$$

where MaxPMF (maximum PeakMF), PMFR (PeakMF rate) and PMFT ( $T$ value for PeakMF) are constants. The greater the PeakMF, the greater the PeakMFRisk, and given the values of the constants in Table 1, PeakMFRisk $=0.04,0.18$, and 0.27 when PeakMF $=40,60$, and $80 \mathrm{~mL} / \mathrm{s}$, respectively.

The combined risk due to udder characteristics and PeakMF (RiskUdd and PeakMFRisk) is

$$
\begin{gathered}
\text { RiskUdd }=\text { Short } T+\text { LowUdd } \\
+ \text { LeakyT + PeakMFRisk }
\end{gathered}
$$

Herd Mastitis Level. Given that mastitis can be either environmental or infectious and also that some types of mastitis pathogens are more virulent than others, the impact of mastitic cows within the herd on the health of an individual cow varies (Bramley and Dodd, 1984; Gröhn et al., 2004). However, in general, the higher is the number of mastitic cows in the herd, the higher the infection pressure on any given cow in the herd (Bramley and Dodd, 1984). The equation for the risk from herd-level mastitis (RiskHerd; see Equation 10) given here allows a general representation of this risk suitable for the situation in which no information about pathogen types is available. Examples of how this might be modified for specific pathogens are given by Østergaard et al. (2005) and Seegers et al. (2003). Risk from herd-level mastitis is derived from 3 components: the relative level of LDH in the herd (HerdRelLDH), the potential for spread because of cow density (Spread), and the longevity of the bacteria (BacLife).

The herd mastitis level is calculated by combining the individual mastitis burdens of the cows in the herd, which are assumed to be reflected in the stable $\mathrm{LDH}$ amounts (Stable). The HerdRelLDH is then calculated as

$$
\text { HerdRelLDH }=\text { HerdMast } / \text { ChronRef }
$$

where HerdMast is the median value of Stable in the herd, and ChronRef is the expected level of LDH caused by chronic mastitis, given the parity and stage of lactation profile of the herd.

Calculating the likelihood for Spread is potentially complicated because it involves considering a number of possible interactions between healthy and infected cows, such as herd size and the proximity of cows, which itself includes stocking density, housing design, and management practices (Østergaard et al., 2005). For simplicity, we have chosen to approximate Spread using a Gompertz function. This recognizes that the underlying relationships are not linear while at the same time providing a value bounded between 0 and 1. Clearly, this aspect of the model could be substantially expanded if users deemed it worthwhile; the current function provides a point of entry for such a development. The function for Spread is

$$
\begin{gathered}
\text { Spread }=\exp \{-\exp [\text { SpreadR }(\text { Herdsize } \\
- \text { SpreadT })]\}
\end{gathered}
$$

where SpreadR and SpreadT are constants (Table 1), and Herdsize is an index of cow density. Under normal housing conditions in a yard (a barn of fixed area), Herdsize would simply be the number of cows. If the average stocking density were changed markedly from the norm (e.g., cows going out to graze), then Herdsize could be the number of animals multiplied by a proportion reflecting the difference in average stocking density. With the constant values given in Table 1 , Herdsize of 50 and 100 result in Spread values of 0.36 and 0.87 , respectively.

The longevity of bacteria is clearly pathogen specific as well as being affected by environmental factors such as local temperature and humidity. If such inputs were readily available, then BacLife could be calculated in a more sophisticated manner than that included in the present model. Given that seasonal variations generally affect most bacteria of importance in the same way (Ribeiro et al., 2001), BacLife reflects the effect of season on bacteria survival (i.e., bacteria survive longer in warm, wet conditions than in cold, dry ones). The longevity of bacteria is a cyclic function of the current date (RunTime), ranging from 0 to 1, calculated as

$$
\begin{aligned}
\text { BacLife }= & 0.5+0.5 \times \text { Hemisphere } \times \cos (2 \Pi \\
& \times \text { DayInYear } / 365)
\end{aligned}
$$

where DayInYear is the number of days between runtime and newyearsday, where newyearsday is the first day of the year. Hemisphere is an offset to adjust the equation to different regions of the globe, with the 
northern hemisphere being -1 and the southern hemisphere being +1 .

The 3 herd components are combined to give mastitis risk caused by RiskHerd, as follows:

$$
\begin{gathered}
\text { RiskHerd }=\text { HerdRelLDH } \times \text { BacLife } \\
\times \text { Spread }
\end{gathered}
$$

where HerdRelLDH is the relative level of $\mathrm{LDH}$ in the herd, BacLife reflects the effect of season on bacteria survival, and Spread is the potential for mastitis spread caused by cow density. The Gompertz function applied to Spread converts it to a 0 to 1 scale.

Current Lactation Disease History. The physiological background for this is the fact that some diseases and disorders often increase the risk of mastitis (Hillerton et al., 1995; Hamann and Krömker, 1997). Several diseases have therefore been included as $A R F$ for mastitis (DisRisk). Two classes of diseases or disorders are recognized: infections and noninfections. The infections are metritis, teat injury, and acidosis, and the noninfections are retained placenta, ketosis, and milk fever. For any given case, the severity of the disease (DisSev), ranging from 0 to 1 , can be input. The severity of the disease could, for example, be grouped in 1 of 3 categories of mild (0.3), average (0.6), or severe $(0.9)$. The constants used in the model when any of these diseases are present are given in Table 2. It is assumed that the effect of a disease is greatest on the day of occurrence and that this effect subsequently declines as a function of days since occurrence (DisDays) to 0 . The function used to calculate the risk of mastitis caused by a given disease (DisRisk) is

$$
\begin{gathered}
\text { DisRisk }=\text { DisSev } \times \text { MaxDis } \times \exp \\
\{-\exp [\text { DisR }(\text { DisDays }- \text { DisT })]\}
\end{gathered}
$$

where Dis- is replaced by the relevant disease name (see Table 2). The period between the model RunTime and date of disease occurrence is DisDays. Because at any one time more than one DisRisk may be in operation, the disease effects are combined such that within each class of disease (infections vs. noninfections), the greatest DisRisk is chosen (i.e., it is assumed that within-class disease risks are not additive). The overall DisRisk is the sum of the highest DisRisk in the infections class and the highest DisRisk in the noninfections class. For each disease, the constant MaxDis gives its effect on the $A R F$ on the day of occurrence. The constants DisR and DisT control the rate of decay of the disease effect with time from occurrence.

Quarter-Level Conductivity. Electrical conductivity has been used as the state variable for detecting mastitis in other models (De Mol et al., 1999). It can therefore be expected to have a reasonable degree of correlation with the amount of $\mathrm{LDH}$, and thus the $M I B R$. Despite this fact, Cond measures (when available) are utilized as part of the $A R F$. This is because Cond has 2 useful attributes: It is inexpensive in terms of cost per measurement, and it is measured at the quarter level. We take advantage of the fact that when it is available, it is usually recorded at every milking and thus can be used to trigger a new LDH measurement (i.e., it can influence $D N S$ ). The main use of quarter-level Cond on $A R F$ is to identify the quarter that is mostly likely to be infected. The input of Cond in the model is an interquarter ratio of the Cond values from a milking. The interquarter ratio is the ratio between the highest and the lowest quarter value within cow and milking. Interquarter ratio has been found to perform better in classifying both clinically and subclinically mastitic cows than the absolute Cond values (Norberg et al., 2004). In recognition of the likely correlation between LDH and Cond, the risk of mastitis due to Cond (RiskCond) is given a relatively low weight in the $A R F$ by utilizing a very high value of an adjusting constant, MaxCond, in the following equation:

$$
\text { RiskCond }=\text { Cond } / \text { MaxCond }
$$

Calculating ARF. The elements that make up the $A R F$, described in the previous sections, combine to give $A R F$ as follows:

$$
\begin{gathered}
A R F=\text { RiskAcc }+ \text { RiskMilkDur }+ \text { RiskUdd } \\
+ \text { RiskHerd }+ \text { DisRisk }+ \text { RiskCond }
\end{gathered}
$$

where RiskAcc is the risk attributable to acceleration in milk yield, RiskMilkDur is the risk attributable to milking duration, RiskUdd is the risk to udder characteristics, RiskHerd is the risk attributable to herd characteristics, DisRisk is the risk of mastitis attributable to other diseases, and RiskCond is the risk attributable to conductivity.

\section{Model Outputs}

The model produces 3 outputs. These are output risk of acute mastitis (AcuteRisk), DNS, and degree of chronic mastitis (ChronDeg).

AcuteRisk. The AcuteRisk presented to the user is generated from the combination of $M I B R$ and $A R F$ as follows:

$$
\text { AcuteRisk }=M I B R+(A R F \times A R F W)
$$


where $M I B R$ is the indicator-based risk and $A R F$ is the risk caused by the additional risk factors. The $A R F W$ is a scaling factor $(=0.25$; Table 1$)$ to weight the $A R F$ relative to the $M I B R$. The current model is not based on the principle that the 2 risks ( $M I B R$ and $A R F$ ) generating AcuteRisk should add up to 1 . The indicator alone should be able to generate a risk of 1 because it can be envisaged that in many situations, no additional information (and therefore no $A R F$ ) is available. The model has been designed to run under such conditions; this also reflects the principle that the indicator is the primary information source in the model. However, because there are well-known predisposing factors for mastitis, the model allows their inclusion as $A R F$ so that susceptible cows are more readily identified when their $\mathrm{LDH}$ levels increase. The weighting (to $25 \%$, i.e., $A R F W=0.25)$ to $A R F$ controls the relative value of this information as opposed to the indicator (i.e., a greater proportion of AcuteRisk should come from MIBR other than $A R F)$. Ultimately, the sum of $M I B R$ and $(A R F \times$ $A R F W$ ) is scaled, making the value of AcuteRisk between 0 and 1.

DNS. This parameter is designed to make the best use of opportunities afforded by automated, real-time, inline sampling technology. It is designed to feed back to the sampling system so that the frequency of milk sampling (i.e., next analysis of $\mathrm{LDH}$ for a particular cow) can be varied according to the calculated risk of acute mastitis. In this scenario, it is desirable for sampling frequency to be increased for a given cow when the risk of mastitis is high and vice versa. Consequently, in the model, an increased risk of acute mastitis causes the $D N S$ to be reduced from a default value (DNSdef). This equation is

$$
\begin{gathered}
\text { DNS = DNSdef } \times(1-\text { MIBR }) \times(1-\text { ARF }) \\
\times(1-\text { signLevChan } \times \text { POutlier }) \\
\times(1-\text { ZM } \times \text { Cond })
\end{gathered}
$$

where $M I B R$ is the indicator-based risk, $A R F$ is the additional risk factor; signLevChan is the sign change in the difference from the current value of Level and the previous value of Level; POutlier is the Kalman filter-derived probability that the current LDH measurement is an outlier, and $Z M$ is an adjustment factor for Cond. Indicator-based risk and $A R F$ are included separately in the equation rather than the combined AcuteRisk value so that if either is high, DNS decreases, regardless of their combined value. In addition to the effects of a high $M I B R$ or $A R F$, if the latest $L D H$ value has a high probability that it is a positive deviation, or outlier, from the normal time series (signLevChan $\times$ POutlier), then another sample should be taken quickly. Also, if there is a high Cond measurement, a follow-up sample should be taken quickly.

ChronDeg. The model also estimates the degree of chronic mastitis. The importance of this lies in the effect of chronic mastitis cases on milk quality. To do this a relative ranking of the cows with respect to ChronDeg is calculated. Because ChronDeg is expected to vary with breed and parity (Harmon, 1994; Sloth et al., 2003), it is calculated relative to a breed-parity reference value to allow comparison between cows across parities. From our own analysis, Chagunda et al. (2006), the relationship between LDH and logSCC was found to be the same across breeds and parities, allowing us to make use of logSCC reference values (logSCCRef) together with reference values for milk yield (RefMY) to generate ChronRef. Degree of chronic mastitis is calculated as

$$
\text { ChronDeg = Stable } / \text { ChronRef }
$$

where ChronRef $=S C C t o L D H c e p t \times R e f M Y+S C C t o L D$ Hslope $\times \log S C C R e f$, and SCCtoLDHcept $=-8.58$ (intercept of LDH vs. logSCC regression), SCCtoLDHslope = 2.38 (slope of LDH vs. $\operatorname{logSCC}$ regression), and $\log S C$ CRef is a breed-parity reference value for the absolute amount of logSCC. The regression coefficients for converting SCC to LDH were derived from Larsen (2003), who used a set of 456 milk samples with both $\mathrm{LDH}$ and SCC available; the values of SCC ranged from 10,000 to $16,097,000$, and the $\mathrm{R}^{2}$ of the regression was $54.3 \%$. The breed-parity RefMY is derived as

$$
R e f M Y=305 \mathrm{~d} M Y \times P a r M Y F a c
$$

where 305d MY is an input representing the expected yield of healthy, well-fed parity 3 cows. ParMYFac for parities 1,2 , and 3 are $0.78,0.94$, and 1 , respectively (Friggens et al., 1999).

\section{MODEL FUNCTIONALITY}

Model functionality was investigated in 2 steps. The first step was to test the logic and robustness of the model. This was done using simulated data. The second step was to validate the model using real data of naturally occurring mastitis from a research herd. A description of this investigation follows.

\section{Testing Model Logic}

Test data were generated for 2 simulated data sets, one representing an acute case of mastitis and the other representing a chronic case of mastitis. Further, addi- 
tional disease incidences were introduced in the chronic mastitis case to test the effect of disease on the $A R F$. The acute mastitis data set was called Tanja, whereas the data set representing a chronic mastitis case was called Anita.

Acute Mastitis Test Data Set (Tanja). The description of this acute mastitis case, which had 2 episodes of increasing LDH, is shown in Figure 3a. The data simulated 2 mastitis occurrences, the first on $\mathrm{d} 16$ and the second on d 33 after calving. From d 2 to 12 after calving, the amount of $\mathrm{LDH}$ was low, ranging from 3 to $37 \mu \mathrm{mol} / \mathrm{min}$. From the second milking of d 12 after calving, LDH increased to $243 \mu \mathrm{mol} / \mathrm{min}$ on d 15 after calving. This was the first episode of an LDH increase. The LDH dropped to about $90 \mu \mathrm{mol} / \mathrm{min}$ on $\mathrm{d} 21 \mathrm{after}$ calving, and the amount stabilized at that level until d 26 after calving. From d 26 after calving, LDH increased again, this time to an even higher level of $311 \mu \mathrm{mol} /$ min on d 31 after calving. Thereafter, LDH dropped again and stabilized around $170 \mu \mathrm{mol} / \mathrm{min}$.

Chronic Mastitis Test Data Set (Anita). The graphical representation of the data set Anita is given in Figure 4a; this data set simulated chronic mastitis. The amount of LDH increased steadily from the second day after calving to about $15 \mathrm{~d}$ after calving. From d 16 after calving, the amount of LDH leveled off, with some marginal increase up to $\mathrm{d} 34$ after calving. After $\mathrm{d} 35$, the amount of LDH decreased slightly up to $\mathrm{d} 40$, when recording stopped. To test the effect of $A R F, 2$ disease incidences-metritis on $\mathrm{d} 12$ and teat injury on $d$ 35-were introduced in the chronic mastitis data set, Anita.

\section{Model Robustness}

To test the robustness of the model, 2 different investigations were conducted using the acute mastitis data set, Tanja. The first investigation aimed at testing the function $D N S$, which allows the model to self-regulate sampling frequency. In this test, the model was run by discarding, at each time step, those $\mathrm{LDH}$ values that occurred between the current model run time (RunTime) and RunTime plus DNS. In other words, LDH data were available only at the times identified by the model for a milk sample to be taken. This is in contrast to initial test runs, which were done using each $\mathrm{LDH}$ data point (i.e., the full time series). The second investigation was aimed at examining the sensitivity of the model to the accuracy of the indicator measurements. This was done by running the model with added random "noise" to the original LDH values in the acute mastitis data set, Tanja. Random values scaled to be in the range $\pm 1,2$, and 3 residual standard deviations (rSD) were used. The rSD was calculated as the standard deviation of the difference between raw LDH amounts and Level in the original data set, Tanja. The rSD was 23.34. For each level of random noise, the model was run 10 times and the average performance was calculated in terms of: number of additional days identified with high mastitis risk, number of "true" cases missed, and delay in time from true case to detection. An arbitrary threshold value of 0.7 for AcuteRisk was used to indicate the onset of a high mastitis risk period.

\section{Model Validation Using Real Data}

Cows and Milk Samples. The data originated from a research farm at the Danish Cattle Research Center, Foulum, Denmark. For the purpose of the current analysis, full lactation profiles of 100 cows (initial data set of 76,257 records from every milking for a period from September 2003 to February 2005) were selected at random. Fifty cows had a mastitis treatment record and the other 50 cows had no mastitis treatment. The cows were from 3 breeds of Danish Holstein, Danish Red, and Danish Jersey. At the farm, all cows were milked with an automatic milking system (3 units, on average $2.3 \pm 0.83$ milkings/cow per d) in which the milk yield was automatically recorded. During each milking, a proportional sample of composite milk was collected automatically from each cow in 10-mL tubes. The tubes contained a concentrated solution of Bronopol (2-bromo-2-nitro-1,0.3 propanediol) to reach $200 \mathrm{ppm}$ (wt/vol) in the filled tube. The automatic milk sampling system was emptied of samples in the morning and in the afternoon. Milk samples were kept at $4^{\circ} \mathrm{C}$ until laboratory analysis, which was done within $24 \mathrm{~h}$ of sampling.

Milk Analysis. In the laboratory, samples were distributed from the $10-\mathrm{mL}$ tubes to 96 -well plates using a Biomek 2000 (Laboratory Automation Workstation, Beckman Coulter, Fullerton, CA) and analyzed for LDH activity in a spectrophotometer/fluorometer (FluoStar, BMG Labtech GmbH, Offenburg, Germany). L-Lactate dehydrogenase activity was analyzed by a fluorometric, kinetic method as described by Larsen (2005) at the Danish Institute of Agricultural Sciences. The accuracies in the laboratory (relative bias) obtained in the present material were 2.7 and $4.9 \%$ for low and high controls, respectively. Intra-assay precision (CV\%) was 8.6 and $3.7 \%$ for low and high controls, respectively, and interassay precision was 15.8 and $10.4 \%$, respectively. The units used in the assay were micromoles per minute per liter. Somatic cell counts were measured at a commercial laboratory (Sønderjysk Kontrolforening, Vojens, Denmark) using Fossomatic 5000 automatic equipment (Foss Electric, Hillerød, Denmark). An example of data from a randomly selected real cow is 
presented in Figure 5a, with the results of the model run presented in Figure 5b.

Definition of Udder Health Status. Cows in the present study were subdivided into healthy and clinically mastitic groups. A healthy cow was based on the cow having no clinical veterinary treatment within the current lactation. For the purpose of the current analysis, to rule out misdiagnosis SCC was used as additional information. Hence, a healthy cow was defined as having no clinical veterinary treatment and having a low SCC (equal to or less than 100,000 cells $/ \mathrm{mL}$ in composite milk). This threshold is in accordance with Laevens et al. (1997), Ma et al. (2000), and Hamann (2005), who indicated that the cell count for composite cow milk should not exceed 100,000 cell $\mathrm{s} / \mathrm{mL}$ for an udder with 4 healthy quarters. The aforementioned definition of a healthy cow was used as the "gold standard" against which specificity was calculated. A clinically mastitic cow was defined as a cow that received veterinary treatment after showing clinical symptoms of mastitis. Clinical mastitis was identified by the farm management staff, confirmed by a veterinarian, and based on clinical signs, including udder inflammation or abnormal milk, with or without general clinical signs (IDF, 1997). In addition, to avoid using data that might have been due to misdiagnosis, a high SCC was used to supplement the classification of mastitic cows in the current analysis. Hence, a cow was defined as clinically mastitic only if it had a veterinary treatment record and also had SCC $\geq 400,000$ cell $/ \mathrm{mL}$. This is in accordance with Hillerton (1999), who pointed out that when the cell count at cow level exceeds 400,000 cell $/ \mathrm{mL}$, the infected quarter has a cell count of $1,000,000$ cells $/ \mathrm{mL}$ or greater, irrespective of a yield depression. A veterinarian following the same protocol as consistently as possible performed all veterinary treatments. The aforementioned definition of a clinically mastitic cow was used as the "gold standard" against which sensitivity was calculated. In this data set, repeat mastitis occurrences recorded within an 8-d period were treated as one occurrence, whereas cases occurring after a gap greater than $8 \mathrm{~d}$ since any previous mastitis record were treated as new mastitis cases. This was done as recommended by the IDF (1997). To concentrate on mastitic periods within the lactation, a 15 - $\mathrm{d}$ window-10 $\mathrm{d}$ before and $5 \mathrm{~d}$ after a mastitis occurrence-was created relative to the recorded mastitis occurrence. If fewer than $3 \mathrm{LDH}$ records existed within the 15-d window, the case was excluded from being used in the model testing.

Model Validation. Three main criteria for testing the model were used: the proportion of healthy cows the model identified as healthy (specificity), the proportion of clinical mastitis cases the model detected as mastitic, and an indication of how early the model de- tected mastitis relative to the treatment records. A threshold of 0.7 for AcuteRisk was used to classify the mastitis risk values generated by the model into negative or positive mastitis detections, allowing calculation of model specificity and sensitivity. Specificity was calculated to identify the proportion of cows the model would classify as being without mastitis (true negatives) within the group of healthy cows (false positives and true negatives). Specificity was calculated as follows:

$$
\begin{aligned}
\text { specificity }= & \text { true negatives } /(\text { false positives } \\
& + \text { true negatives })
\end{aligned}
$$

Sensitivity (the proportion of mastitic cows detected by the model) was calculated within the mastitic group as follows:

$$
\begin{aligned}
\text { sensitivity } & =\text { true positives } / \text { (true positives } \\
& + \text { false negatives })
\end{aligned}
$$

Along with the values of specificity and sensitivity, predictive values were calculated as either the proportion of true positives among the apparent positives, or the proportion of true negatives among the apparent negatives. However, it is important to note that the model does not actually produce mastitis indications in terms of positive and negative indications but rather produces a continuous risk of mastitis. The threshold used here was merely for model testing purposes. To determine how early the model would detect mastitis, the first rise of AcuteRisk beyond 0.7 within each mastitis window was taken to be the earliest time at which the model detected the mastitis incidence. Other threshold values of 0.6 and 0.8 were also tested.

\section{RESULTS}

\section{Simulated Data}

The model responded in a biologically logical way. As can be seen from Figures $3 \mathrm{~b}, 4 \mathrm{~b}$, and $4 \mathrm{c}$, risk of mastitis increased as the amount of $\mathrm{LDH}$ increased above the baseline LDH level (Stable) and decreased as LDH returned to baseline levels. Number of days to next sampling decreased when the risk of mastitis increased, whereas $D N S$ increased with reducing risk. For example, in Figure 3b (data set Tanja), the model indicated a high risk of mastitis from d 10 to about d 15 after calving, as shown by high values of AcuteRisk. With this increase in AcuteRisk was an associated decrease in DNS. These results indicate that the model outputs responded as expected according to the changing profile 
a
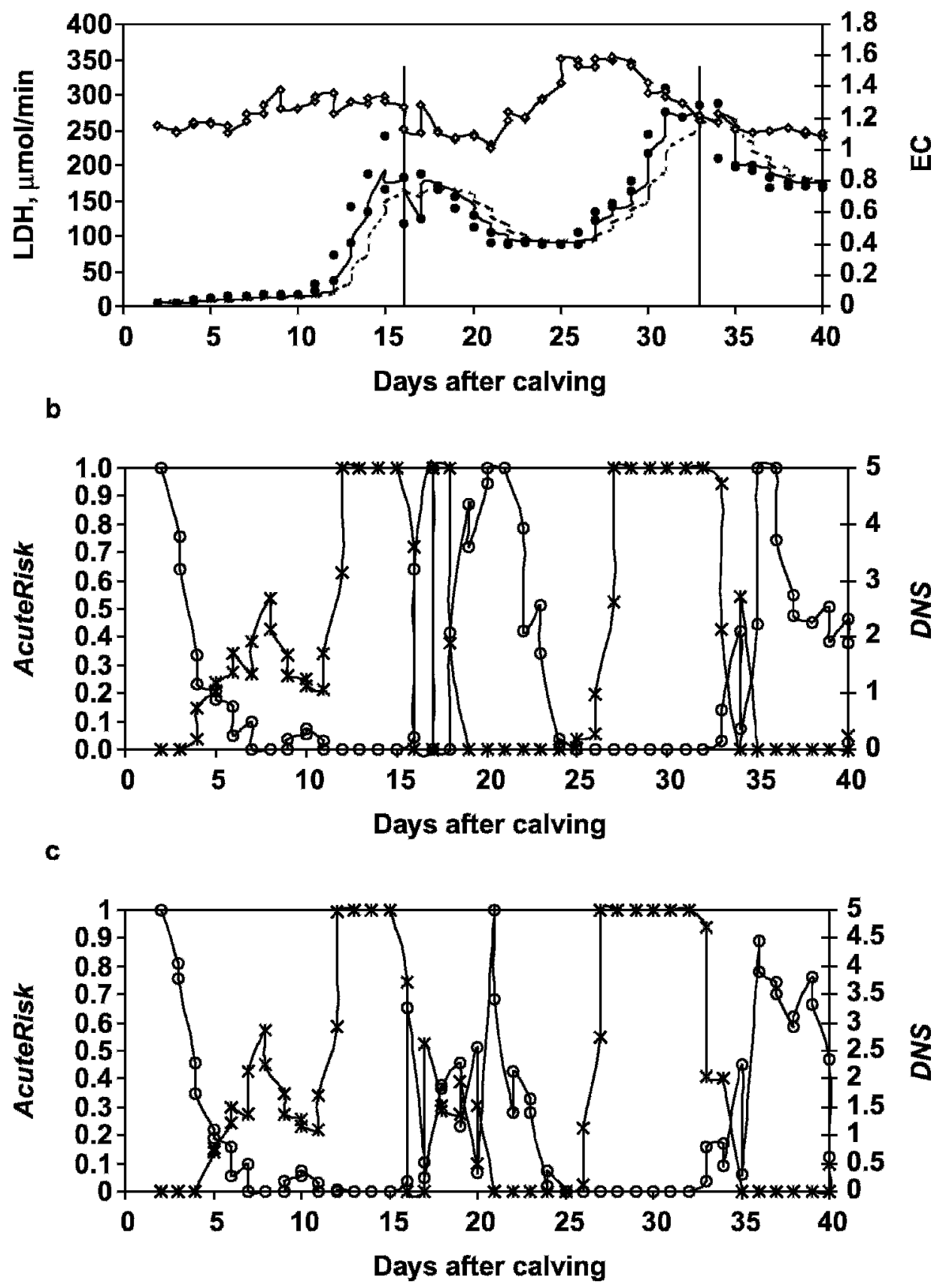

Figure 3. (a) Simulation of an acute mastitis case with 2 episodes of increased amounts of L-lactate dehydrogenase (LDH). Raw values for the amounts of LDH are shown as dots (-); smoothed values of LDH (Level), calculated using an extended Kalman filter, are shown by the solid line (-). The baseline 7-d average value of the smoothed amount of LDH (Stable) is shown as a broken line (----). The vertical lines indicate the 2 incidences of mastitis that were simulated in the data set on d 16 and 33 after calving. The solid line with open diamonds $(\diamond)$ indicates the electrical conductivity (EC) values calculated as the interquarter ratio (secondary axis). This data set is referred to as Tanja. (b) Results after running the biological model based on the simulated data set Tanja, as illustrated in Figure 3a. AcuteRisk [solid line with asterisk (*) plotted on the primary $y$-axis] indicates the risk of the cow developing mastitis, with risk ranging from 0 to 1 . Days to next sample $[D N S$; solid line with open circles $(\bigcirc)$ plotted on the secondary $y$-axis] is an output from the model indicating when the next sample of LDH should be taken. (In this model run, the DNS function is not used to regulate the sampling frequency.) (c) Results after running the biological model based on the simulated data set Tanja using the DNS function, which allows the model to self-regulate sampling frequency. AcuteRisk [solid line with asterisks (*) plotted on the primary $y$-axis] indicates the risk of the cow developing mastitis, with risk ranging from 0 to 1 . DNS [solid line with open circles $(\bigcirc)$ plotted on the secondary $y$-axis] is an output from the model indicating when the next sample of LDH should be taken. 
a
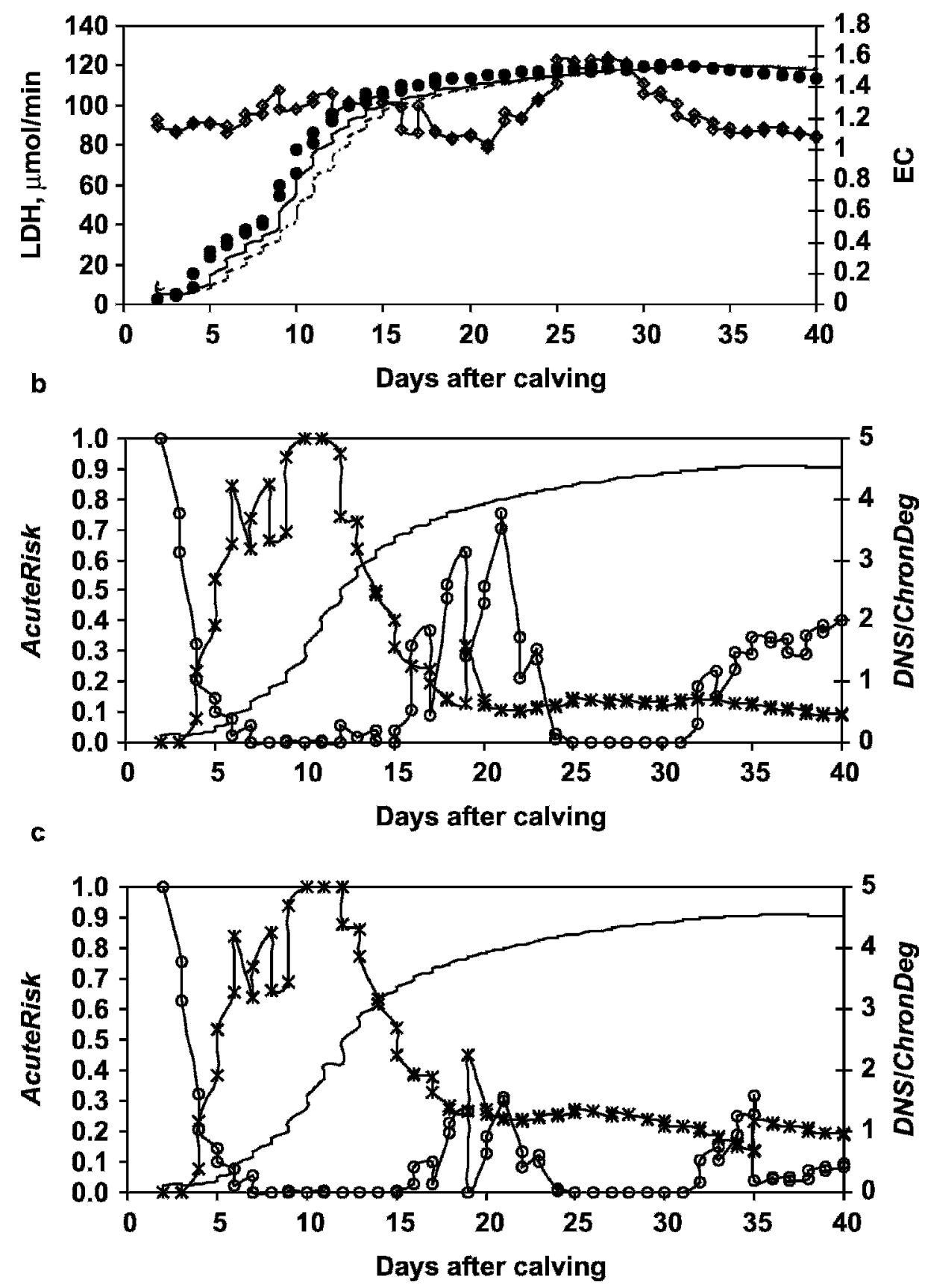

Figure 4. (a) Simulation of a chronic mastitis case with a gradual increase in the amount of L-lactate dehydrogenase (LDH; $\bullet$ ). Smoothed values of LDH calculated using an extended Kalman filter (Level) are shown as a solid line (-). The baseline 7-d average value of the smoothed LDH amount (Stable) is shown as a broken line (--). The solid line with open diamonds $(\diamond)$ indicates the electrical conductivity (EC) values calculated as interquarter ratio (secondary axis). This data set is referred to as Anita. (b) Results from running the biological model based on the simulated data set Anita, as illustrated in Figure 4a. AcuteRisk [solid line with asterisks $(*)$ plotted on the primary $y$ axis] indicates the risk of the cow developing acute mastitis, with risk ranging from 0 to 1 . Days to next sample [DNS; solid line with open circles $(\mathrm{O})$, also plotted on the secondary $y$-axis] is an output from the model indicating when the next sample of LDH should be taken. ChronDeg [ $\mu \mathrm{mol} / \mathrm{min}$; solid line (-) plotted on the secondary $y$-axis and scaled down by a factor 7 to fit into the same axis with $D N S$ ] shows the degree of chronic mastitis. (c) Results from running the biological model based on the simulated data set Anita with the addition of 2 incidences of other diseases, metritis on d 18 and teat injury on d 35 after calving. AcuteRisk [solid line with asterisks (*) plotted on the primary $y$-axis] indicates the risk of the cow developing mastitis, with risk ranging from 0 to 1 . DNS [solid line with open circles (O), also plotted on the secondary $y$-axis] is an output from the model indicating when the next sample of LDH should be taken. ChronDeg [solid line (-) plotted on the secondary $y$-axis and scaled down by a factor of 7 to fit into the same axis with DNS] shows the degree of chronic mastitis. 
Table 3. Consequences of adding random variation (noise) to the raw LDH amount on the performance of the biological model ${ }^{1}$

\begin{tabular}{|c|c|c|c|}
\hline \multirow[b]{2}{*}{ Evaluation criteria } & \multicolumn{3}{|c|}{ Range of random variation (no. of rSD) } \\
\hline & 1 & 2 & 3 \\
\hline Extra risk periods detected (AcuteRisk $>0.7)$ & 1.1 & 4.7 & 8.8 \\
\hline Deviation in days to onset of high risk before a defined mastitis case & 0.3 & 0.4 & -0.8 \\
\hline
\end{tabular}

\footnotetext{
${ }^{1}$ Levels of noise equivalent to $+1,2$, and 3 residual standard deviations (rSD) of the original data were added. Values presented are
} averages over 10 sets of random noise relative to the original data set (Tanja) with no added noise.

of LDH and consequently identified the 2 simulated acute mastitis incidences on $\mathrm{d} 16$ and 33 after calving. It is worth noting that on $\mathrm{d} 24$ after calving, although AcuteRisk was low, DNS dropped. This is because on that day there was an increase in the Cond value (Figure 3a). From d 35 after calving, AcuteRisk was relatively low and $D N S$ was notably higher than 0 . Degree of chronic mastitis, which is not shown in Figure 3, had a shape similar to Level.

Results from the model runs using the DNS function, which allowed the model to self-regulate sampling frequency, are presented in Figure 3c. When the model was run to self-regulate the sampling frequency, results were similar to those when the model was run using a full data set. Using the DNS function, the model identified the same mastitis cases as in the full data set but used only $72 \%$ of the number of "samples" in the full time series.

The results of the model runs on the data set representing a chronically mastitic cow (Anita) indicated that the model was able to identify not only acute cases of mastitis but also chronic ones. In Figure 4 b, one can see that the model initially increased the AcuteRisk as a result of the persistently increasing amount of $\mathrm{LDH}$ (as shown by the ChronDeg line) relative to the rising baseline (Stable) up until d 12, after which it gradually dropped, even though $\mathrm{LDH}$ was still increasing. Although the simulated case was not an incidence of acute mastitis, the initial rate of increase of $\mathrm{LDH}$ was sufficient for the model to return a high AcuteRisk value. Degree of chronic mastitis persistently increased to about d 25 after calving and remained high thereafter, indicating an increasing risk of chronic mastitis. As expected, DNS decreased with increased AcuteRisk and increased otherwise.

When there was an input of 2 diseases (metritis and teat injury) in the chronic mastitis case, the results indicated an increased AcuteRisk and a reduced DNS. On 12 and 35 after calving (Figure 4c), which were the days when these diseases were registered, the disease effect in both cases pushed the AcuteRisk up for some days after the disease registration. As can be noted in Figure 4c, metritis had a short and not-so-prominent effect on AcuteRisk, whereas teat injury had a notice- ably higher and more persistent effect. This was expected, because in the model constants (Table 2), teat injury had higher values than metritis (i.e., MaxDis of 0.9 vs. 0.5 and $D i s R$ of 21 vs. 10).

The consequences of adding random variation (noise) to the $\mathrm{LDH}$ values are presented in Table 3 . When 1 rSD of noise was added, the model indicated an average of 1.1 extra periods in which the acute rise exceeded 0.7 . The extra mastitis risk periods increased by an average of 4.7 and 8.8, with noise levels of 2 and $3 \mathrm{rSD}$, respectively. However, the model did not miss any of the 2 true mastitis incidences, with noise levels of 1 and $2 \mathrm{rSD}$. When $3 \mathrm{rSD}$ of noise were added to the amount of $\mathrm{LDH}$, the model missed on average 0.1 of the true mastitis cases. With increasing noise in the $\mathrm{LDH}$ values, there was a relatively small shift $(0.4 \mathrm{~d})$ in days to detection of mastitis up to $2 \mathrm{rSD}$ added noise. At the highest level of noise addition ( $3 \mathrm{rSD}$ ), there was a numerically significant delay $(-0.8 \mathrm{~d})$ in detection of true mastitis cases. The interassay precision for the low control for analysis of real LDH values in the laboratory was $15.8 \%$. Over the equivalent range in $\mathrm{LDH}$, the simulated noise from adding $1 \mathrm{rSD}$ equated to a coefficient of variation of $27.4 \%$. This implies that the model is robust to a level of noise substantially greater than expected from analysis of uncertainties in real LDH data.

\section{Real Data}

Based on the validation set of LDH obtained from a dairy herd under an automatic milking system from 50 healthy cows and 50 cows with at least one recorded mastitis treatment, the sensitivity and specificity of the model for mastitis detection were calculated. In the healthy cow subgroup, 19,015 records were identified as true negatives and 94 records were identified as false positives. In the clinically mastitic cow subgroup, 404 records were identified as true positives and 88 records were false negatives. The specificity was $99 \%$, with a predictive value of $97 \%$. Changing the threshold of AcuteRisk from 0.7 to 0.6 and 0.8 did not yield numerically significant changes in specificity values. The ability of the model to detect clinical mastitis (sensitivity) 
a
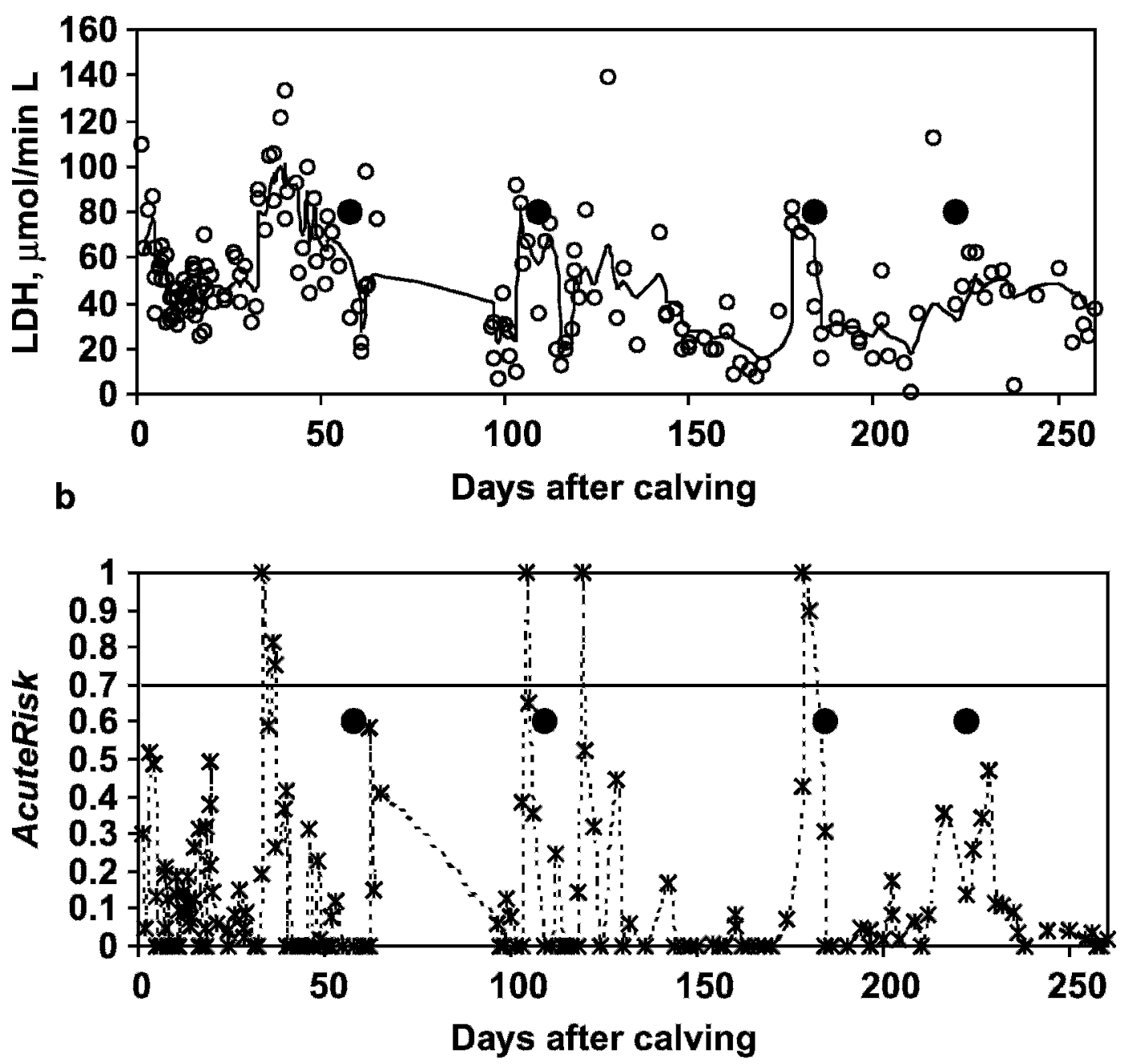

Figure 5. (a) An example (randomly selected) of real data for a cow with naturally occurring mastitis depicting model inputs: amount of L-lactate dehydrogenase [LDH, $\mu \mathrm{mol} / \mathrm{min}$; open circles $(\bigcirc)]$; the smoothed values of $\mathrm{LDH}$ (Level, $\mu \mathrm{mol} / \mathrm{min}$ ), calculated using an extended Kalman filter, in the biometrical model [solid line (-)]; and mastitis treatment (large solid dots (@)]. (b) Results from a model run based on the data of a cow with naturally occurring mastitis (panel a) indicating AcuteRisk [broken line with asterisk (*)] and recorded mastitis occurrence [solid dots $(\bullet)$ ]. An arbitrary threshold of 0.7 (horizontal line) was used to classify AcuteRisk into positive and negative modelgenerated identifications of mastitis.

was $82 \%$, with a predictive value of $98 \%$. Changing the threshold of AcuteRisk to 0.6 and 0.8 resulted in sensitivity values of 78 and $82 \%$, respectively. Descriptive statistics for the number of days between model detection of mastitis (i.e., AcuteRisk $\geq 0.7$ ) and treatment records for the real data of naturally occurring mastitis are presented in Table 4 . The results indicate that the model was able to detect mastitis on average $3.5 \mathrm{~d}(\mathrm{SD}=3.99 \mathrm{~d})$ earlier with respect to the recorded mastitis treatment. Further, the results showed that during the 15-d window around the mastitis treatment date, AcuteRisk outputs from the model were higher (mean $=0.20, \mathrm{SD}=0.32$ ) than in the normal periods of lactation outside the mastitis window $($ mean $=0.09$, $\mathrm{SD}=0.17$.

\section{DISCUSSION}

The current study aimed to develop a biological model for detecting mastitis in individual cows in a dairy herd based on real-time measurements of an indicator in milk. This goal was achieved. The integration of risk factors and mastitis indicators in a biologically meaningful way offers potential as a useful decision support tool for dairy farmers that allows early detection of individual cases of mastitis in the herd. The model, 
which is diagnostic in nature, was able to identify mastitis progression in data sets designed to simulate acute and chronic mastitis cases. The model was able to distinguish between a plateau chronic case and a steadily increasing chronic case by producing different levels of risk for mastitis. The performance of the model was not affected by the reduction in the number of data points through use of the DNS function. The model identified the same mastitis risk periods when compared with the run with the full data set. The calculation of DNS is an important feature of the model because it takes advantage of the opportunities afforded by automated online sampling technology. Thus, sampling frequency can be increased at times when there is a high risk of mastitis and decreased when there is little risk of mastitis. Distribution of samples in this way increases the efficiency of early detection of mastitis and offers economic advantages.

When random noise was introduced into the indicator values, the model was robust to an amount of variation that was greater than the noise expected from the interassay variation for the measurement used here, $\mathrm{LDH}$ (see also Bogin et al., 1976; Harmon, 1994). The model was stable in identifying the "true" mastitis cases, although the onset of a high mastitis risk period before a defined mastitis case increased by $0.36 \mathrm{~d}$ with $2 \mathrm{rSD}$ added noise (Table 3 ).

The results of the model runs based on real data and using LDH as the indicator in milk indicate that the model is able to detect true mastitis incidences and is also able to correctly identify cows that do not have mastitis. On average, the model detected mastitis 3.5 $\mathrm{d}$ earlier than the day of treatment. Using a threshold of 0.7 for the model output risk, the specificity from the current model (99\%) was higher than those reported by Hillerton (2000) of 70\% for temperature and $95 \%$ for a combination of Cond, temperature, and milk yield, but was similar to that reported by De Mol and Ouweltjes

Table 4. Descriptive statistics for model-generated risk of mastitis (AcuteRisk) relative to recorded mastitis treatment

\begin{tabular}{|c|c|c|c|}
\hline \multirow[b]{2}{*}{ Statistic } & \multirow{2}{*}{$\begin{array}{l}\text { Days between } \\
\text { treatment and } \\
\text { model mastitis } \\
\text { detection }^{1}\end{array}$} & \multicolumn{2}{|c|}{ AcuteRisk value ${ }^{2}$} \\
\hline & & $\begin{array}{l}\text { Within } \\
\text { mastitis } \\
\text { window }\end{array}$ & $\begin{array}{l}\text { Out of } \\
\text { mastitis } \\
\text { window }\end{array}$ \\
\hline $\mathrm{n}$ & 427 & 857 & 21,354 \\
\hline Mean & -3.46 & 0.20 & 0.09 \\
\hline SD & 3.99 & 0.32 & 0.17 \\
\hline Min & -9.68 & 0 & 0 \\
\hline Max & 4.25 & 1 & 1 \\
\hline
\end{tabular}

${ }^{1}$ Model mastitis detection was defined as the first rise of AcuteRisk beyond 0.7 relative to mastitis treatment.

${ }^{2}$ Mastitis window was a 15 -d period comprising $10 \mathrm{~d}$ before and 5 $\mathrm{d}$ after mastitis treatment.
(2001). The sensitivity values of $82 \%$ for clinical mastitis obtained from the current model are generally higher than those reported previously. For example, De Mol and Ouweltjes (2001) reported sensitivities of between 66 and $67 \%$ from a model based on Cond. Hillerton (2000) reported sensitivities in automatic systems for the following indicators: NAGase, $60 \%$; Cond between 70 and $80 \%$; temperature, $50 \%$; milk yield between 20 and 40\%; and combined Cond, temperature, and milk yield between 85 and $90 \%$. Although failure to detect about $20 \%$ of true acute cases in absolute terms and also the variation of AcuteRisk within and outside the mastitis window would be problematic in commercial situations, one major focus of the current model is on early warning and allowing a range of different strategies according to a continuous scale of risks. The gain in this case is that the model generates AcuteRisk based on repeated measurements, hence indicating a trend in the direction of real-time mastitis risk as opposed to a one-off measurement of the mastitis indicator. However, it is important to note that the test reported here was carried out on one farm and remains to be validated in the field across several farms. Nevertheless, the fact that the current results were obtained from a farm under an automatic milking system, which is normally associated with a high degree of variation, indicates that the current model offers promising prospects in the more stable environment of conventional milking systems.

The specificities and sensitivities obtained depend on the criteria applied for classifying a mastitis treatment record as "true" mastitis or not. This indicates the importance of recording precision for reference values in specificity or sensitivity testing. Further, the values obtained also depend on the value of model output risk chosen as the threshold for classifying these risks as mastitis or not. As illustrated by Norberg et al. (2004), altering the threshold allows widely varying values of specificity and sensitivity to be obtained. The limitations of this form of test are clear, especially when one considers that mastitis infection is not a binomial trait but rather a continuous one because both the infection pressure and degree of response are on continuous scales. For these reasons, we have chosen not to present the model output as "alarms" but rather to present the output as a continuous risk ranging from 0 to 1 . We believe that this provides the end user with more information (which can then be collapsed into alarms should the end user wish).

The current model was illustrated using $\mathrm{LDH}$ as the main indicator measured in milk. Several studies have shown that the activity of LDH in individual milk samples has proved to be an important indicator of bovine mastitis (e.g., Larsen, 2005; Chagunda et al., 2006). 
As biosensor assays for enzymes like LDH in milk are now becoming available, they provide an opportunity for automated, real-time, inline mastitis detection. Although this is the case, within the current architecture the model could be implemented with any mastitis indicator measured in milk. The design of the model to incorporate $A R F$ would allow several indicators to be combined. Indeed conductivity is used in this way. However, when combining information or indicators, care must be taken to avoid double counting and contraindications. Only a limited number of the risk factors for mastitis described in the literature were included in the model as $A R F$. There were 2 reasons for this: We were trying to achieve the simplest adequate description, and we wanted to avoid double counting caused by correlations between risk factors. Both are important for achieving robust and widely applicable models. For example, traits such as the lifetime number of mastitis incidences have previously been used as a single measure of the cow's susceptibility to mastitis. However, mastitis is both an infectious and an environmental disease. Thus, to some extent we can expect the lifetime number of mastitis incidences to reflect the disease pressure placed on the cow by the environments she has been in. Lifetime number is therefore the result not only of that cow's susceptibility, but also a number of other factors that are not inherent to that cow. For this reason, lifetime number was not chosen as a predictor of the cow's susceptibility even though in the herd where this model was being used, it had the practical advantage of being automatically generated if the risk of mastitis output was used to generate disease incidences. Other factors that have been suggested to increase the risk of mastitis (Harmon, 1994; Peeler et al., 2000) were not included in the model. One such example is negative energy balance. It is expected that poor energy status will, among other things, increase the risk of mastitis. However, energy balance is difficult to measure in practice, and the literature evidence is not clear-cut for an effect of energy status independent of other effects with which it is correlated in epidemiological-type studies. We therefore chose instead to use MYAcc as an indicator of physiological stress (Kronfeld, 1976; Knight et al., 1999; Ingvartsen et al., 2003), which is both biologically relevant and easy to apply in practice. The list of other diseases included as mastitis risk factors is also not exhaustive. Some other diseases (e.g., lameness) have not been included in the current model. This is mainly because of a lack of information in the literature on the direct influence of such diseases on mastitis. However, the indirect influence of lameness on mastitis through milking duration and milking interval has been accommodated in the model. Further, the architecture of the model allows the inclusion of other diseases once information about their direct influence on mastitis is available.

Any model is always a trade-off between including all known effects in a complex model and including fewer, readily available inputs in a simpler model. By including a number of optional inputs of biological risk factors, we believe this model can be adapted to a wide range of circumstances. Despite making extensive use of the literature, we expect that the values of some of the constants presented in this paper will need adjusting to optimize the performance of the model. The model is not expected to perform equally well in all circumstances. In particular, differences could be expected in, for example, infection pressure when different mastitis pathogens are dominant in different localities. Further, various mastitis agents differ in their pathophysiological pathways; thus, the model may behave differently depending on the mastitis-causing agent. The results obtained could therefore differ if bacteria-specific mastitis types were considered, and the extent to which this is so should be quantified by further research. Given the built-in flexibility of the model, we expect that it could easily be extended to deal with such cases. Because the model utilizes biological "rules" and logic in its underlying architecture, we believe it is an improvement on existing mastitis models and will make a useful contribution to dairy cattle management. An indication of the costs-benefits of a system such as the one described in the current paper would be informative on the practicalities of such systems. However, this is beyond the scope of the current paper, which focuses on model description and functionality. Economic implications of such a system have been covered by Østergaard et al. (2005). The current model is one of a set, which includes reproduction (Friggens and Chagunda, 2005) and ketosis detection models (Nielsen et al., 2005).

\section{CONCLUSIONS}

We have described a model for early detection of individual cow mastitis based on measurements of an indicator in milk and incorporating known biological risk factors of mastitis susceptibility. The results of runs with simulated data suggest that the model has the potential to provide the basis for a useful decision support tool for mastitis management. This conclusion is heavily supported by the analysis of real data using $\mathrm{LDH}$ as the indicator in milk, where sensitivity was greater than $80 \%$ and specificity was greater than $99 \%$.

\section{ACKNOWLEDGMENTS}

The contributions of Inge Korsgaard, who developed and carried out the extended Kalman filter embedded 
in this model, and Carsten Ridder, who developed the software and test tools for the model, are highly appreciated. Many thanks to Martin Bjerring for the farm data and Karin V. Østergaard for help in preparing the manuscript. This study was funded by a grant financed by the Directorate for Food, Fisheries and Agri Business, Lattec I/S, Danish Cattle Association, and the Danish Institute of Agricultural Sciences. The comments and suggestions of 4 anonymous referees are greatly appreciated.

\section{REFERENCES}

Baxter, E. S., P. M. Clarke, F. H. Dodd, and A. S. Foot. 1950. Factors affecting the rate of machine milking. J. Dairy Res. 17:117-127.

Bogin, E., G. Ziv, and J. Avidar. 1976. Enzyme activities in normal and inflamed bovine udder tissues. Zentralbl. Veterinaermed. A 23:460-466.

Bogin, E., G. Ziv, J. Avidar, B. Rivetz, S. Gordin, and A. Saran. 1977. Distribution of lactate dehydrogenase isoenzymes in normal and inflamed bovine udders and milk. Res. Vet. Sci. 22:198-200.

Bramley, A. J., and F. H. Dodd. 1984. Review on the progress of dairy science: Mastitis control-Progress and prospects. J. Dairy Res. 51:481-512.

Chagunda, M. G. G., T. Larsen, M. Bjerring, and K. L. Ingvartsen. 2006 . L-Lactate dehydrogenase and $N$-acetyl- $\beta$-D-glucosaminidase activities in bovine milk as indicators of clinical mastitis. J. Dairy Res. (accepted)

Deluyker, H. A., S. N. Van Oye, and J. F. Boucher. 2005. Factors affecting cure and somatic cell count after Pirlimycin treatment of subclinical mastitis in lactating cows. J. Dairy Sci. 88:604614.

de Mol, R. M., A. Keen, G. H. Kroeze, and J. M. F. H. Achten. 1999. Description of a detection model for oestrus and diseases in dairy cattle based on time series analysis combined with a Kalmar filter. Comp. Electr. Agric. 22:171-185.

de Mol, R. M., and W. Ouweltjes. 2001. Detection model for mastitis in cows milked in an automatic milking system. Prev. Vet. Med. 49:71-82.

de Mol, R. M., and W. E. Woldt. 2001. Application of fuzzy logic in automated cow status monitoring. J. Dairy Sci. 84:400-410.

France, J., and J. H. M. Thornley. 1984. Mathematical Models in Agriculture. Butterworths, London.

Fricke, P. M. 2002. Scanning the future-Ultrasonology as a reproductive management tool in dairy cattle. J. Dairy Sci. 85:1918-1926.

Friggens, N. C., and M. G. G. Chagunda. 2005. Prediction of the reproductive status of cattle on the basis of milk progesterone measures: Model description. Theriogenology 64:155-190.

Friggens, N. C., G. C. Emmans, and R. F. Veerkamp. 1999. On the use of simple ratios between lactation curve coefficients to describe parity effects on milk production. Livest. Prod. Sci. 62:1-13.

Frost, A. R., C. P. Schofield, S. A. Beaulah, T. T. Mottram, J. A. Lines, and C. M. Wathes. 1997. A review of livestock monitoring and the need for integrated systems. Comp. Electr. Agric. 17:139-159.

Giesecke, W. H., W. H. Gerneke, and I. B. J. van Rensburg. 1972. The morphology of the bovine teat canal. J. S. Afr. Vet. Assoc. 43:351-354.

Gröhn, Y. T., D. J. Wilson, R. N. Gonzalez, J. A. Hertl, H. Schulte, G. Bennett, and Y. H. Schukken. 2004. Effect of pathogen-specific clinical mastitis on milk yield in dairy cows. J. Dairy Sci. 87:3358-3374.

Hamann, J. 1987. Effect of machine milking on teat end conditionA literature review. In Machine Milking and Mastitis. Pages 33-39 in International Dairy Federation (IDF) Bulletin no. 215, Brussels, Belgium.
Hamann, J. 2005. Diagnosis of mastitis and indicators of milk quality. Pages 82-90 in Proc. 4th Intl. Dairy Fed. (IDF) Mastitis Conf., Mastitis in Dairy Production: Current Knowledge and Future Solutions. H. Hogeveen, ed. Wageningen Academic Publ., Wageningen, The Netherlands.

Hamann, J., and M. Duck. 1984. Modification of milking systems in relations to the prevention of mastitis. Milchwissenschaft $39: 12-15$.

Hamann, J., and V. Krömker. 1997. Potential of specific milk composition variables for cow health management. Livest. Prod. Sci. 48:201-208.

Harmon, R. J. 1994. Physiology of mastitis and factors affecting somatic cell counts. J. Dairy Sci. 77:2103-2112.

Hillerton, J. E., A. J. Bramley, R. T. Staker, and C. H. McKinnon. 1995. Patterns of intramammary infection and clinical mastitis over a 5-year period in a closely monitored herd applying mastitis control measures. J. Dairy Res. 62:39-50.

Hillerton, J. E. 1999. Redefining Mastitis Based on Somatic Cell Count. Pages 4-6 in Bulletin 345. International Dairy Federation, Brussels, Belgium.

Hillerton, J. E. 2000. Detecting Mastitis Cow-Side. Proc. 39th Mtg. Natl. Mastitis Council, Atlanta, GA. Natl. Mastitis Counc., Verona, WI.

IDF. 1997. Recommendations for Presentation of Mastitis-Related Data. Bulletin 321. International Dairy Federation, Brussels, Belgium.

Ingvartsen, K. L., R. J. Dewhurst, and N. C. Friggens. 2003. On the relationship between lactation performance and health: Is it yield or metabolic imbalance that causes production diseases in dairy cattle? A position paper. Livest. Prod. Sci. 83:277-308.

Kato, K., K. Mori, and N. Katoh. 1989. Contribution of leucocytes of the origin of lactate dehydrogenase isozymes in milk of bovine mastitis. Jpn. J. Vet. Sci. 51:530-539.

Kitchen, B. J., G. Middleton, I. G. Durwar, R. J. Andrews, and M. C. Salmon. 1980. Mastitis diagnostic tests to estimate mammary gland epithelial cell damage. J. Dairy Sci. 63:978-983.

Knight, C. H., D. F. Beever, and A. Sorensen. 1999. Metabolic load to be expected from different genotypes under different systems. Br. Soc. Anim. Sci. Occ. Publ. 24:27-35.

Korsgaard, I. R., and P. Løvendahl. 2002. An introduction to multiprocess class II mixture models. Proc. 7th World Congr. Genet. Appl. Livest. Prod., Montpellier, France.

Kronfeld, D. 1976. The potential importance of the proportions of glucogenic, lipogenic and amonigenic nutrients in regard to the health and productivity of dairy cows. Adv. Anim. Physiol. Nutr. $7: 5-26$.

Laevens, H., H. Deluyker, Y. H. Schukken, L. De Meulemeester, R. Vandermeersch, E. De Muelenaere, and A. De Kruif. 1997. Influence of parity and stage of lactation on the somatic cell count in bacteriologically negative dairy cows. J. Dairy Sci. 80:3219-3226.

Larsen, T. 2005. Determination of lactate dehydrogenase (LDH) activity in milk by a fluorometric assay. J. Dairy Res. 72:209216.

Larsen, T. 2003. Samstilling af analyser for lactate dehydrogenase, $N$-acetyl- $\beta$-D-glucosaminase og somatic cell count på mælkeprøver. Internal Report. Danish Institute of Agricultural Sciences, Research Centre Foulum, Foulum, Denmark. 7 pp. (in Danish).

Lucy, M. C. 2001. Reproductive loss in high-producing dairy cattle: Where will it end? J. Dairy Sci. 84:1277-1293.

Ma, Y., C. Ryan, D. M. Barbano, D. M. Galton, M. A. Rudan, and K. J. Boor. 2000. Effects of somatic cell count on quality and shelf-life of pasteurized fluid milk. J. Dairy Sci. 83:264-274.

Mottram, T., M. Velasco-Garcia, P. Berry, P. Richards, J. Ghesquiere, and L. Masson. 2002. Automatic on-line analysis of milk constituents (urea, ketones, enzymes and hormones) using biosensors. Comp. Clin. Pathol. 11:50-58.

Nielsen, N. I., N. C. Friggens, M. G. G. Chagunda, and K. L. Ingvartsen. 2005. Predicting risk of ketosis in dairy cows using in-line measurements of $\beta$-hydroxybutyrate in milk: A biological model. J. Dairy Sci. 88:2441-2453. 
Norberg, E., H. Hogeveen, I. R. Korsgaard, N. C. Friggens, K. H. M. N. Sloth, and P. Løvendahl. 2004. Electrical conductivity of milk: Ability to predict mastitis status. J. Dairy Sci. 87:10991107.

Østergaard, S., M. G. G. Chagunda, N. C. Friggens, T. W. Bennedsgaard, and I. C . Klaas. 2005. Stochastic simulation modeling of pathogen specific mastitis control in a dairy herd. J. Dairy Sci. 88:4243-4257.

Peeler, E. J., M. J. Green, J. L. Fitzpatrick, K. L. Morgan, and L. E. Green. 2000. Risk factors associated with clinical mastitis in low somatic cell count British dairy herds. J. Dairy Sci. 83:2464-2472.

Ribeiro, A. R., F. Garino, Jr., J. A. B. Silva, E. T. Watanabe, C. R. Valle, and E. O. Costa. 2001. Seasonality on the occurrence of environmental bovine mastitis. Napgama 4:10-14.
Seegers, H., C. Fourichon, and F. Beaudeau. 2003. Production effects related to mastitis and mastitis economics in dairy cattle herds. Vet. Res. 34:475-491.

Smith, A. F. M., and M. West. 1983. Monitoring renal transplants: An application of the multiprocess Kalman filter. Biometrics 39:867-878

Sloth, K. H. M. N., N. C. Friggens, P. Løvendahl, P. H. Andersen, J. Jensen, and K. L. Ingvartsen. 2003. Potential for improving description of bovine udder health status by combined analysis of milk parameters. J. Dairy Sci. 86:1221-1232.

Woolford, M. W., J. H. Williamson, and H. V. Henderson. 1998. Changes in electrical conductivity and somatic cell count between milk fractions from quarters subclinically infected with particular mastitis pathogens. J. Dairy Res. 65:187-198. 